\title{
The role of 5-alpha reductase inhibitors in prostate pathophysiology: Is there an additional advantage to inhibition of type 1 isoenzyme?
}

\author{
Larry Goldenberg, OBC, MD, FRCSC;* Alan So, MD, FRCSC; ${ }^{\dagger}$ Neil Fleshner, MD, MPH, FRCSC,; \\ Ricardo Rendon, MD, ${ }^{\ddagger}$ Darrel Drachenberg, MD, FRCSC; ** Mostafa Elhilali, MD, FRCSC ${ }^{\dagger \dagger}$
}

\begin{abstract}
Normal growth and function of the prostate are contingent on the reduction of testosterone to dihydrotestosterone (DHT) by 5 -alpha reductase (5-AR) enzymes types 1 and 2. It has been theorized that an overabundance of DHT may be implicated in the pathogenesis of both benign prostatic hyperplasia (BPH) and prostate cancer. Inhibitors of 5-AR such as dutasteride and finasteride may therefore have an important role in the prevention and treatment of $\mathrm{BPH}$ and prostate cancer. Dutasteride provides greater suppression of DHT than finasteride, thereby underlying the hypothesis that inhibition of both type 1 and type 2 would provide correspondingly greater protection than inhibition of type 2 alone. We review the potential significance of the 5-AR inhibitors in reducing the risk of prostate cancer according to the basic biology of prostate disease
\end{abstract}

Can Urol Assoc J 2009;3(3Suppl2):S109-14

\section{Introduction}

$\mathrm{O}$ pportunities for studying the molecular action of androgens emerged in the 1960s, when radioactive compounds suitable for experimentation were initially introduced. In 1968, Bruchovsky and Wilson ${ }^{1,2}$ and Anderson and Liao $^{3}$ reported that dihydrotestosterone is the active intracellular form of testosterone, and receptors for dihydrotestosterone were demonstrated for the first time. Two isoforms of the enzyme have been identified: type 1, which is found in skin, prostatic epithelium and to a lesser extent in stroma, and type 2, which predominates in prostatic stromal tissue. Normal growth and function of the prostate are contingent on the reduction of testosterone to dihydrotestosterone (DHT) by 5 -alpha reductase (5-AR).

Overabundance of DHT has been implicated in the pathogenesis of benign prostatic hyperplasia (BPH) and prostate cancer. Preventing DHT synthesis via 5-AR inhibition has been shown to have a remarkable effect on benign prostatic disease with low toxicity. ${ }^{4}$ Thus, there is much interest in the potential role for 5-AR inhibitors (5-ARIs) in the management of prostatic intraepithelial neoplasia (PIN) and the prevention of prostate cancer. ${ }^{5}$ This report reviews current knowledge about the role of androgens and the 5-AR system in prostate disease and highlights their significance in reducing the risk of prostate cancer.

\section{Prostate dependence on androgens}

Androgens play an essential role in prostatic development and function, but are also involved in prostate disease pathogenesis. ${ }^{6}$ Without androgens, the prostate would not develop and the gland would atrophy if androgen support was withdrawn. ${ }^{5}$ The principal and functionally active androgen DHT is synthesized from testosterone by 5 -AR types 1 and $2{ }^{6,7}$ This leads to a 5 -fold higher concentration of DHT v. testosterone within the prostate cells, $v$. an 11-fold higher concentration of testosterone in the blood. ${ }^{4}$ Free testosterone diffuses directly into the epithelial or stromal cells of the prostate, where it is converted rapidly and irreversibly into DHT by the 5-AR system positioned on the nuclear membrane. DHT binds to the androgen receptor with high affinity, and this complex, mediated by coregulators such as NCOA3, is transported into the nucleus. Once inside, this newly formed complex binds to androgen-responsive elements, initiates transcription and ultimately controls the regulation of the cell cycle, cell growth and differentiation.?

5-AR has the biologically important role of being able to concentrate intraprostatic DHT when/if serum testosterone levels are physiologically low. As serum testosterone levels increase, there is less of this need of 5-AR to help accumulate androgen in the prostate. ${ }^{8}$ In a study by Wright and colleagues, ${ }^{8}$ SpragueDawley rats were castrated for 2 weeks to allow their prostates to fully regress and were then given testosterone implants of various sizes in the presence or absence of a 5-AR inhibitor. Dramatic increases in prostate weight were seen even at the lowest serum testosterone levels in the absence of 5-AR inhibition, while significantly higher serum testosterone levels were needed to achieve prostate growth in the 
presence of 5-AR inhibition. ${ }^{8}$ The difference in potency of testosterone $\mathrm{v}$. DHT in relation to prostate growth is small compared with intraprostatic androgen concentrations, but is much greater if serum testosterone levels are considered. ${ }^{8}$

A critical question regarding androgen action is whether testosterone and DHT are responsible for regulating the expression of different androgen-response genes in the prostate. Experimental studies suggest that testosterone is more potent than DHT in stimulating the expression of many androgen-response genes in the regressed prostate. ${ }^{9}$ The expression of these androgenresponse genes is likely to be associated with androgen-dependent and prostate-specific differentiation, including the very important preservation of the capacity of prostatic cells to undergo apoptosis, and less associated with stimulating proliferation during prostate growth. ${ }^{9}$ On the other hand, high levels of intracellular DHT result in cellular proliferation and delay in cellular differentiation, an important fact when considering

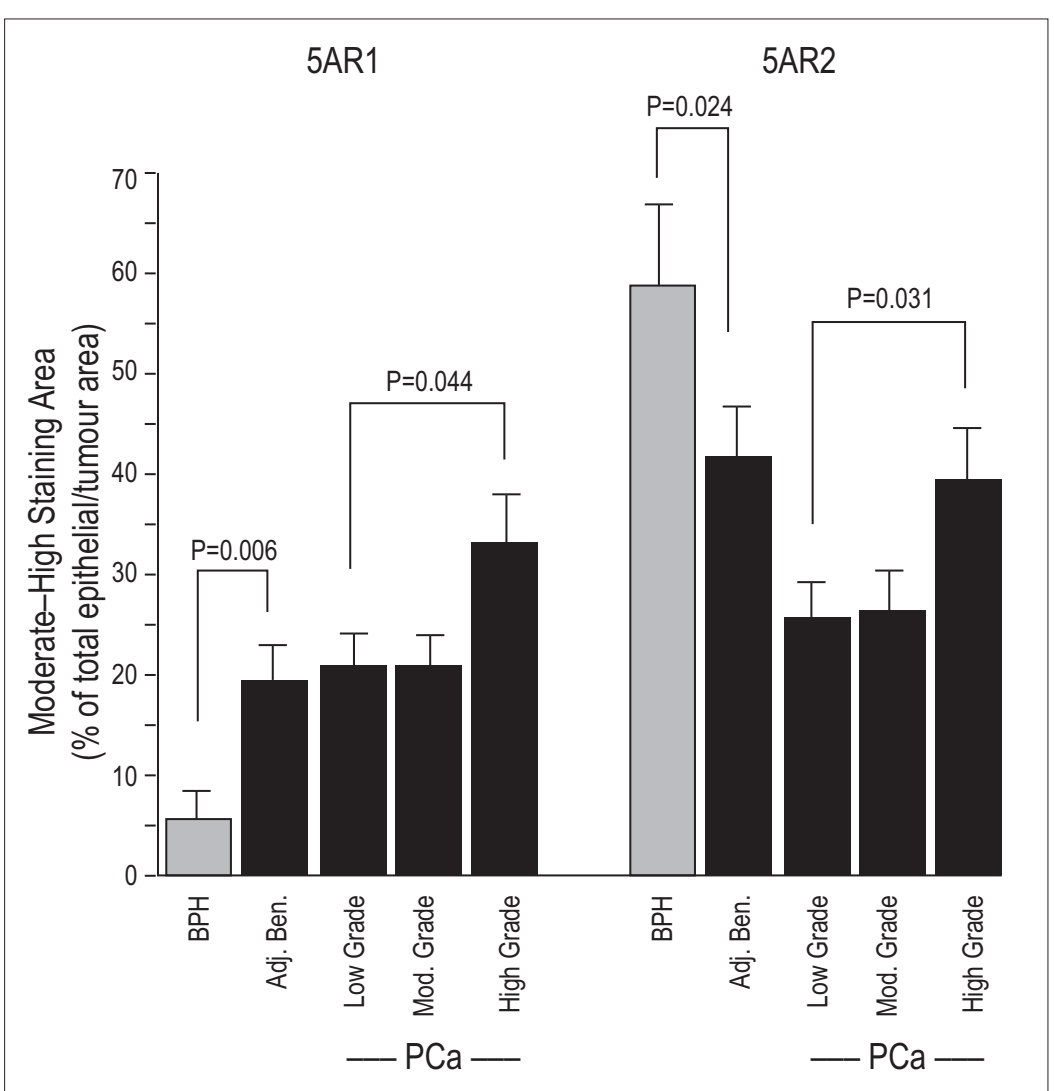

Fig. 1. Mean area of moderate- to high-intensity staining, as a percentage of total epithelial or tumour area, for 5-AR1 and 5-AR2 in benign prostatic hyperplasia (BPH), benign tissues adjacent to cancer (Adj. Ben.), and low-, moderate- or high-grade cancer. Reproduced with permission from the Journal of Urology.13 treatment of early prostatic disease, or premalignant conditions with 5-AR inhibition.

\section{5-ARs and prostate cancer development}

5-AR2 has traditionally been viewed as the most clinically relevant of the 5-AR isoenzymes because of its high concentration in prostatic tissue and its greater affinity to bind testosterone than $5-A R 1 .{ }^{6}$ However, more recently, 5-AR1 expression has also been shown to be increased in prostate cancer compared with normal and BPH tissue at both the messenger RNA (mRNA) level ${ }^{10,11}$ and at the protein level. ${ }^{10,12}$ Thomas and colleagues provide a review of this data. ${ }^{6}$ lehlé and colleagues ${ }^{11}$ have shown that the expression of 5-AR1 mRNA was significantly increased $(p<0.05)$ in prostate cancer compared with normal and BPH tissue but that the level of 5-AR2 mRNA was not statistically different from that observed in normal prostate. ${ }^{11}$ By contrast, the increased expression of 5-AR1 mRNA in BPH tissue was not statistically different from that of type 2 in the same tissue." Similarly, Thomas and colleagues ${ }^{10}$ showed that 5 -AR1 mRNA levels are increased, whereas 5-AR2 mRNA levels are decreased, in primary prostate cancer compared with normal and BPH tissues. Thomas and colleagues ${ }^{13}$ also showed by immunostaining that 5 -AR1 is increased in adjacent benign tissues, lowgrade prostate cancer and moderate-grade prostate cancer compared with that in BPH. 5-AR1 was even further increased in high-grade cancers compared with $\mathrm{BPH}$, or low- and moderate-grade cancers (Figure 1). ${ }^{13}$ By contrast, staining for 5-AR2 was decreased in adjacent benign tissue, as well as in low- and moderate-grade cancers compared with that in $\mathrm{BPH}$, and was increased in high-grade cancers compared with low-grade cancers (Figure 1). ${ }^{13}$ A recent study by $\mathrm{Xu}$ and colleagues ${ }^{14}$ reported that 5-AR2 enzymatic activity was more than 10 -fold higher than 5-AR1 enzymatic activity in both normal rat ventral prostatic cells and normal human prostate tissue. However, prostate cancer cells from both humans and rats expressed low levels of 5$A R 2$, and enhanced levels of 5-AR1 activity compared with nonmalignant tissues. ${ }^{14}$ Thomas and colleagues have demonstrated that levels of both 5-AR1 and 5-AR2 are increased in localized high$\mathrm{v}$. low-grade prostate cancer and that the levels of 5-AR1 were higher in benign tissue adjacent to cancer than in BPH. The mean (+ SEM) area of moderate-to-high staining for 5-AR1 increased from $20.5 \%(3.7 \%)$ in low-grade cancers (Gleason score 
$5-6)$ to $32.9 \%(5.3 \%)$ in high-grade cancers (Gleason score 8-10) $(p=0.0439$, high v. low grade)..$^{13}$ Staining in 21 moderate-grade (Gleason score 7) cancers was the same as in low-grade cancers. ${ }^{13}$ Therefore, an important point to consider is that 5-AR1, based on its increased expression in all prostate cancer tissue except for $\mathrm{BPH}$, and especially its predominance in higher grades of cancer, may play an important role in premalignant and malignant tissues. This generates the hypothesis that the inhibition of both isoenzymes would be more effective for prevention and possibly treatment of prostate cancer than inhibition of 5-AR2 alone in man. ${ }^{6}$

\section{5-ARs as targets for prevention and management}

Dutasteride and finasteride are both 5-ARIs available for use in the management of $\mathrm{BPH} .{ }^{15}$ Finasteride is a potent inhibitor of 5-AR2 (IC50 $69 \mathrm{nmol} / \mathrm{L}$ ) but much less effective at inhibiting 5-AR1 (IC50 $360 \mathrm{nmol} / \mathrm{L}$ ). By contrast, dutasteride has been proven to competitively inhibit both 5-AR1 and 5-AR2 (IC50 $6 \mathrm{nmol} / \mathrm{L}$ and $7 \mathrm{nmol} / \mathrm{L}$, respectively) and to a greater degree than finasteride. ${ }^{6}$ Thus the question arises: Does a dual inhibitor of 5-AR outperform that of a single inhibitor?

\section{DHT suppression}

$\mathrm{Xu}$ and colleagues ${ }^{14}$ reported that dutasteride, but not finasteride (even at a dose of $72 \mathrm{mg} / \mathrm{kg}$ per day), suppressed DHT content and inhibited Dunning $\mathrm{R}-3327 \mathrm{H}$ prostate cancer growth in non-castrated male rats. Interestingly, when rats were treated with dutasteride, prostate cancer growth was significantly reduced, but not to the same extent as with castration, which eliminates testosterone, ${ }^{14}$ thereby supporting the notion that although testosterone is not as potent as DHT it still can stimulate cellular metabolism and malignant growth to a certain extent. $\mathrm{Xu}$ and colleagues also reported that although daily oral treatment with finasteride $(70 \mathrm{mg} / \mathrm{kg}$ per day, twice a day) significantly reduced the growth of $\mathrm{LNCaP}$ human prostate cancer xenografts in non-castrated male nude mice, the reduction was not as dramatic as that by equimolar dosing with dutasteride $(100 \mathrm{mg} / \mathrm{kg}$ per day, twice a day). The fact that dutasteride reduced growth to a greater extent than finasteride in both intact and castrated mice further supports the importance of type 1 inhibition. ${ }^{14}$
Results of a phase II, double-blind, placebocontrolled, comparative dose-ranging trial clearly showed that serum DHT suppression was significantly greater with dutasteride $(94.7 \% \pm 3.3 \%$ with $0.5 \mathrm{mg})$ than with finasteride $(70.8 \pm 18.3 \%, p<$ $0.001)$, and that the response to dutasteride was less variable. Serum DHT concentrations returned to within $20 \%$ of baseline values in only 4 weeks post-treatment in patients given finasteride, compared to 16 weeks in those given dutasteride (Figure 2). ${ }^{16}$ From the same study, Roehrborrn and colleagues ${ }^{17}$ reported greater than $90 \%$ DHT suppression in $85.4 \%$ of patients treated with dutasteride $(0.5 \mathrm{mg})$ and in only $2.2 \%$ of patients treated with finasteride (5 mg). By comparison, greater than $70 \%$ DHT suppression was observed in $100 \%$ of the dutasteride-treated patients and in $49 \%$ of the finasteride-treated patients..$^{17,18}$ In another approach, Gleave and colleagues ${ }^{19}$ showed that men who were treated with dutasteride (doses $0.5 \mathrm{mg}$ daily or higher) for 4 months prior to radical prostatectomy had significantly lower intraprostatic DHT concentrations but had higher testosterone concentrations than in untreated prostatectomies. Furthermore, men who received dutasteride had lower tumour volumes, without significant changes in treatment alteration scores or atrophy, than those who received surgery alone. ${ }^{19}$ Similarly, Iczkowski and colleagues ${ }^{20}$ showed that in cancer tissues from men who received pre-surgical treatment with dutasteride compared with that from men treated with finasteride, tumour volume had decreased significantly (mean $15 \%$ v. $24 \%, p=0.025$ ), the percentage

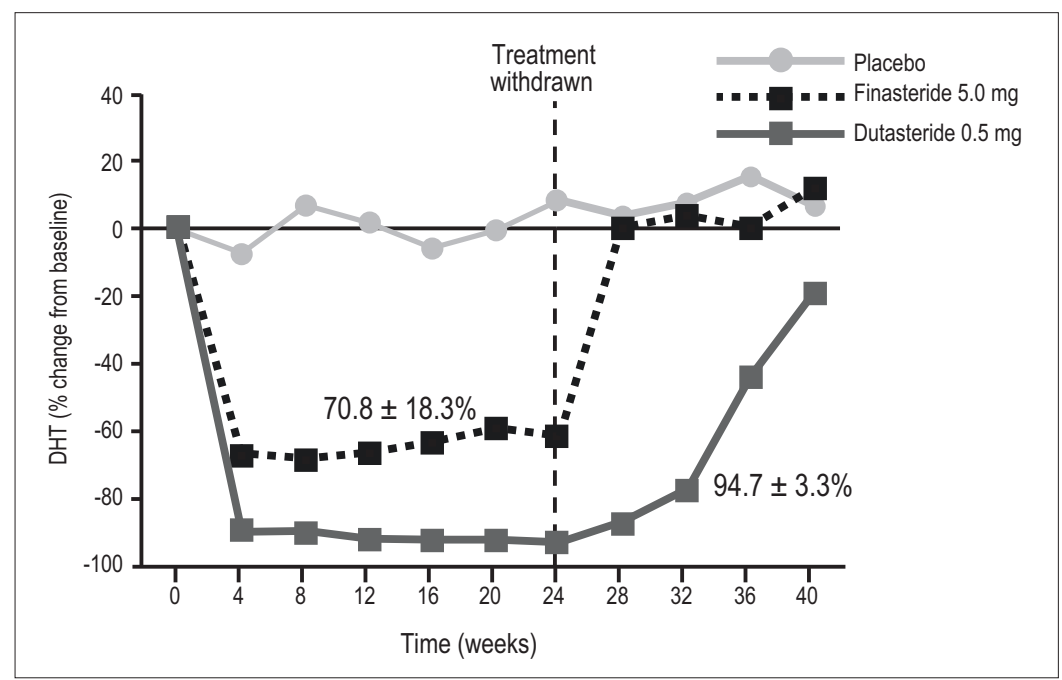

Fig. 2. Dual 5-alpha reductase inhibition results in greater serum dihydrotestosterone (DHT) suppression. Percentage changes from baseline in DHT over time are shown as arithmetic means. Data were derived from the Journal of Clinical Endocrinology and Metabolism. ${ }^{16}$ 
of atrophic epithelium had increased ( $p=0.041)$ and both the stroma/gland ratio and treatment alteration effect had doubled. The ability to induce phenotypic alterations in both the benign and neoplastic prostate suggest a potential chemopreventive role for dutasteride. ${ }^{20}$

\section{Effects on serum PSA concentration}

Prostate specific antigen (PSA) is a biomarker whose expression is governed by androgens, and whose serum concentration can be used in the detection of prostate cancer. That said, treatment with 5-ARIs such as finasteride or dutasteride results in decreased serum PSA concentrations, which may make the interpretation of the test difficult. ${ }^{21}$ In the randomized, double-blind, active-controlled Enlarged Prostate International Comparative Study (EPICS), dutasteride and finasteride treatment resulted in similar median PSA suppression of approximately $55 \%$ at 12 months (GlaxoSmithKline, data on file). Finasteride treatment in the PLESS trial resulted in a median change of $57 \%$ after 4 years. ${ }^{22}$ Marks and colleagues, ${ }^{23}$ in a review of 3 large-scale, phase III studies, reported that dutasteride treatment results in a median change in PSA of $66 \%$ at 4 years. Differences in PSA suppression between finasteride and dutasteride may reflect the impact of blocking 5-AR1 in prostate cells. That PSA is not reduced to zero implies ongoing binding of testosterone on the androgen receptor, which normally is much weaker than DHT binding, ${ }^{24}$ or the possible existence of a type 3 isoenzyme. ${ }^{25}$ The significance of these data is highlighted by the results of the Prostate Cancer Prevention Trial (PCPT), in which the overall decrease in prostate cancer cases associated with finasteride treatment was coupled with an increase in the number of high-grade prostate cancer cases. ${ }^{26}$ Thirty-seven percent of cancers in the finasteride group had a Gleason score of 7 to 10 compared with $22 \%$ in the placebo group, with the increase in high-grade cancers present mainly in the Gleason 8 to 10 range..$^{26}$ It has been suggested that detection bias for high-grade tumours in patients treated with finasteride may help to explain these findings and that this may be due in part to improved specificity and sensitivity of detection of PSA and DRE under 5-ARI treatment. ${ }^{27}$ Finasteride inhibits only 5-AR2, resulting in a decrease in PSA of $50 \%$. In low-grade cancer, the amount of 5-AR2 may still predominate; therefore, both finasteride and dutasteride should produce an adequate reduction in the level of PSA. By contrast, in high-grade cancers, levels of 5-AR1 and 5-AR2 are both increased ${ }^{13}$ so that finasteride will theoretically block progression of low-grade cancers but not high-grade cancers. This issue may be clarified with the release of the Reduction by DUtasteride of Prostate Cancer Events (REDUCE) study data. ${ }^{28}$

\section{Influence of somatic 5-AR mutations}

The identification and characterization of somatic 5-AR mutations in human prostate cancer tissue have implications both in the predisposition and in the progression of prostate cancer. Makridakis et al. reported that in about $60 \%$ of prostate tumours, somatic substitutions occur in the protein-coding region of the SRD5A2 gene, which encodes 5-AR. Approximately $20 \%$ of tumour samples had SRD5A2 mutations that significantly activated steroid androgen receptor, while only 7\% had SRD5A2 mutations that reduced enzyme activity significantly. It is, therefore, possible that these genetic variants may play an important role in the development and progression of BPH and prostate cancer, and may help individualize cancer treatment and prevention through pharmacogenetic mechanisms. ${ }^{29}$ Further in vitro analysis showed that dutasteride was an efficient steroid 5-AR inhibitor for most of the enzyme variants, with the exception of P48R and F194L, and that dutasteride treatment would also be expected to result in lower pharmacogenetic variation in vivo than finasteride treatment. ${ }^{30}$

\section{Castration-resistant prostate cancer}

Castration-resistant prostate cancer (CRPC) remains a therapeutic challenge, and it has been hypothesized that dual 5-AR inhibition may be valuable in its management. Eggener and colleagues ${ }^{31}$ reported that a combination of dutasteride, but not finasteride, plus castration, achieved significantly greater reductions in $\mathrm{LNCaP}$ tumour growth in xenograft-bearing nude mice than castration alone $(p<0.05)$ and that dutasteride-treated mice were 3 to 5 times more likely to be alive 70 days following treatment than those treated with finasteride. Current data suggest that the androgen receptor is expressed and continues to mediate androgen signaling after failure of androgen deprivation therapy. Although hormone suppression treatment eliminates the detectable levels of testosterone in the blood, the levels in tissue remain high enough to stimulate androgen receptors and allow both survival of tumour cells and induction of resistance by overex- 
pression of the receptor. ${ }^{32,33}$ Locke and colleagues ${ }^{34}$ have shown that all enzymes necessary for androgen synthesis are expressed in prostate cancer tumours, and some seem to be up-regulated during CRPC progression, suggesting that de-novo synthesis may be a driving force behind castration resistance. A combination of androgen deprivation (goserelin with bicalutamide and/or ketoconazole) and dual inhibition (dutasteride) could inhibit tumour growth by reducing intracellular androgen synthesis, and this is currently being studied at the University of Washington. ${ }^{33}$

\section{Conclusions}

The enzyme 5-AR, which reduces testosterone to DHT, exists in 2 forms: type 1 and type 2 . Dutasteride inhibits both, whereas finasteride inhibits type 2 only. Available data show that dutasteride, in contrast to finasteride, provides greater suppression of DHT than finasteride, is able to shrink tumour volume quicker and more dramatically than finasteride, and is more effective against genetic variants of 5-AR than is finasteride. Thus, the dual inhibition of 5-AR may prove more useful in the treatment of $\mathrm{BPH}$ in reducing the risk of prostate cancer and in the treatment of CRPC. Full clinical investigations of these and other approaches to the inhibition of 5-AR are ongoing and results are greatly anticipated.

*Professor and Head, Department of Urologic Sciences, University of British Columbia Vancouver, BC; 'Assistant Professor, Department of Urologic Science, University of British Columbia, Vancouver, BC; ; Head, Division of Urology, University Health Network, Toronto, ON; ‘Associate Professor, Department of Urology, Dalhousie University, Halifax, NS; ${ }^{\star \star}$ Assistant Professor and Director of Research, Department of Surgery, Section of Urology, University of Manitoba, Winnipeg, Manitoba; ${ }^{\text {tH} H e a d, ~ D e p a r t m e n t ~}$ of Surgery, McGill University, Montréal, QC.

This article has been peer reviewed.

Competing interests: None declared.

\section{References}

1. Bruchovsky N, Wilson JD. The conversion of testosterone to 5-alpha-androstan-17beta-0-3-one by rat prostate in vivo and in vitro. J Biol Chem 1968;243:2012-21.

2. Bruchovsky N, Wilson JD. The intranuclear binding of testosterone and 5-alpha-androstan17-beta-0l-3-one by rat prostate. J Biol Chem 1968;243:5953-60.

3. Anderson KM, Liao S. Selective retention of dihydrotestosterone by prostatic nuclei. Nature 1968;219:277-9.

4. Hudak SJ, Hernandez J, Thompson IM. Role of 5 alpha-reductase inhibitors in the management of prostate cancer. Clin Interv Aging 2006; 1:425-31.

5. Imamoto T, Suzuki H, Yano M, et al. The role of testosterone in the pathogenesis of prostate cancer. Int J Urol 2008;15:472-80.
6. Thomas LN, Douglas RC, Lazier CB, et al. Type 1 and type 25 alpha-reductase expression in the development and progression of prostate cancer. Eur Urol 2008;53:244-52.

7. Chu LW, Reichardt JK, Hsing AW. Androgens and the molecular epidemiology of prostate cancer. Curr Opin Endocrinol Diabetes Obes 2008;15:261-70.

8. Wright AS, Douglas RC, Thomas LN, et al. Androgen-induced regrowth in the castrated rat ventral prostate: role of 5alpha-reductase. Endocrinology 1999;140:4509-15.

9. Dadras SS, Cai X, Abasolo I, et al. Inhibition of 5alpha-reductase in rat prostate reveals differential regulation of androgen-response gene expression by testosterone and dihydrotestosterone. Gene Expr 2001;9:183-94.

10. Thomas LN, Lazier CB, Gupta R, et al. Differential alterations in 5alpha-reductase type 1 and type 2 levels during development and progression of prostate cancer. Prostate 2005;63:231-9.

11. Iehlé C, Radvanyi F, Gil Diez de Medina S, et al. Differences in steroid 5alpha-reductase iso-enzymes expression between normal and pathological human prostate tissue. J Steroid Biochem Mol Biol 1999;68:189-95.

12. Titus $\mathrm{MA}$, Gregory $\mathrm{CW}$, Ford OH $3 \mathrm{rd}$, et al. Steroid 5alpha-reductase isozymes I and II in recurrent prostate cancer. Clin Cancer Res 2005;11:4365-71.

13. Thomas LN, Douglas RC, Lazier CB, et al. Levels of 5alpha-reductase type 1 and type 2 are increased in localized high grade compared to low grade prostate cancer. J Urol 2008;179:147-51.

14. Xu Y, Dalrymple SL, Becker RE, et al. Pharmacologic basis for the enhanced efficacy of dutasteride against prostatic cancers. Clin Cancer Res 2006;12:4072-9.

15. Rittmaster RS. 5alpha-reductase inhibitors in benign prostatic hyperplasia and prostate cancer risk reduction. Best Pract Res Clin Endocrinol Metab 2008;22:389-402.

16. Clark RV, Hermann DJ, Cunningham GR, et al. Marked suppression of dihydrotestosterone in men with benign prostatic hyperplasia by dutasteride, a dual 5 alpha-reductase inhibitor. J Clin Endocrinol Metab 2004;89:2179-84.

17. Roehrborn C, Ray P, Gittelmann M, et al. The novel dual 5 alpha-reductase inhibitor, dutasteride, is effective and well-tolerated for benign prostatic hyperplasia in black men. [poster 635]. European Urology Association Annual Meeting; Madrid; $12-15$ March, 2003

18. Djavan B, Mirone V. The benefits of dual inhibition of 5 alpha reductase. Eur Urol Suppl 2006;5:1013.

19. Gleave M, Qian J, Andreou C, et al. The effects of the dual 5alpha-reductase inhibitor dutasteride on localized prostate cancer-results from a 4-month pre-radical prostatectomy study. Prostate 2006;66:1674-85.

20. Iczkowski KA, Qiu J, Qian J, et al. The dual 5-alpha-reductase inhibitor dutasteride induces atrophic changes and decreases relative cancer volume in human prostate. Urology 2005;65:76-82.

21. Lilia H, Ulmert D, Vickers AJ. Prostate-specific antigen and prostate cancer: prediction, detection and monitoring. Nat Rev Cancer 2008;8:268-78.

22. Andriole GL, Guess HA, Epstein II, et al. Treatment with finasteride preserves usefulness of prostate-specific antigen in the detection of prostate cancer: results of a randomized, double-blind, placebo-controlled clinical trial. PLESS Study Group. Proscar Longterm Efficacy and Safety Study. Urology 1998;52:195-201.

23. Marks LS, Andriole GL, Fitzpatrick JM, et al. The interpretation of serum prostate specific antigen in men receiving 5alpha-reductase inhibitors: a review and clinical recommendations. J Urol 2006;176:868-74.

24. Grino PB, Griffin JE, Wilson JD. Testosterone at high concentrations interacts with the human androgen receptor similarly to dihydrotestosterone. Endocrinology 1990;126:1165-72.

25. Uemura M, Tamura K, Chung S, et al. Novel 5 alpha-steroid reductase (SRD5A3, type-3) is overexpressed in hormone-refractory prostate cancer. Cancer Sci 2008;99:81-6.

26. Thompson IM, Goodman PJ, Tangen CM, et al. The influence of finasteride on the development of prostate cancer. N Engl J Med 2003;349:215-24.

27. Akduman B, Crawford ED. The PCPT: New findings, new insights, and clinical implications for the prevention of prostate cancer. Eur Urol Suppl 2006;5:634.

28. Musquera M, Fleshner NE, Finelli A, et al. The REDUCE trial: chemoprevention in prostate cancer using a dual 5alpha-reductase inhibitor, dutasteride. Expert Rev Anticancer Ther 2008;8:1073-9.

29. Makridakis N, Akalu A, Reichardt JK. Identification and characterization of somatic steroid 5alpha-reductase (SRD5A2) mutations in human prostate cancer tissue. Oncogene 2004;23:7399-405.

30. Makridakis N, Reichardt JK. Pharmacogenetic analysis of human steroid 5 alpha reductase type II: comparison of finasteride and dutasteride. J Mol Endocrinol 2005:34:617-23. 
Goldenberg et al.

31. Eggener SE, Stern JA, Jain PM, et al. Enhancement of intermittent androgen ablation by "off-cycle" maintenance with finasteride in LNCaP prostate cancer xenograft model. Prostate 2006;66:495-502.

32. Scher HI, Buchanan G, Gerald W, et al. Targeting the androgen receptor: improving outcomes for castration-resistant prostate cancer. Endocr Relat Cancer 2004:11:459-76.

33. Maximal suppression of the androgen axis in clinically localized prostate cancer (NCT00298155). http://www.clinicaltrials.gov/c†2/show/NCT00298155?term= nct002981558rank=1. Accessed January 16, 2009.
34. Locke JA, Guns ES, Lubik AA, et al. Androgen levels increase by intratumoral de novo steroidogenesis during progression of castration-resistant prostate cancer. Cancer Res 2008;68:6407-15.

Correspondence: Dr. Larry Goldenberg, Diamond Healthcare Centre, 2775 Laurel Street Level 6, Vancouver, BC V5Z 1M9; I.gold@ubc.ca

\section{We welcome your comments on the journal and on specific articles.}

All letters will be considered for publication in the journal.

Send your letters to the Editor-in-Chief at journal@cua.org

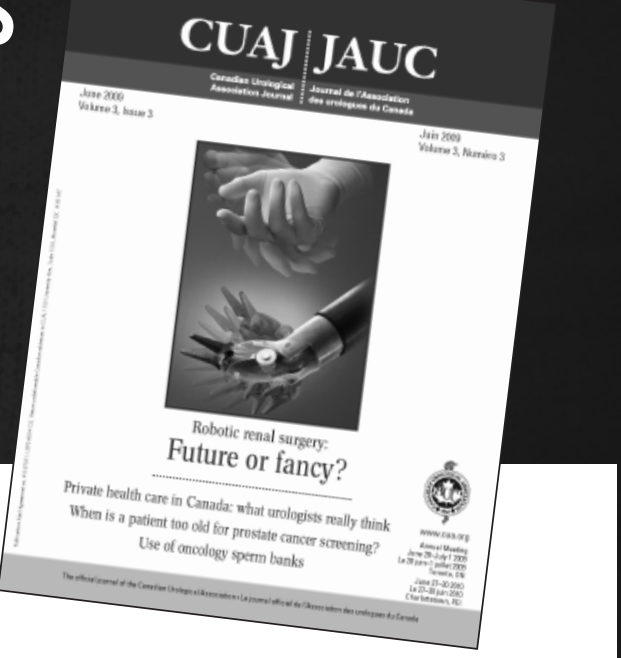

